International Mathematical Forum, 1, 2006, no. 16, 763-768

\title{
Semi-Invariant Product of A Certain Class of Almost Contact Manifolds
}

\author{
Falleh R. Al-Solamy \\ Department of Mathematics, King AbdulAziz University \\ P. O. Box 80015, Jeddah 21589 \\ Saudi Arabia \\ falleh@hotmail.com
}

\begin{abstract}
We prove that a semi-invariant submanifold $M$ of a Kenmotsu space form $\bar{M}(c)(c \neq-1)$ is a semi-invariant product if every geodesic in $M$ is a circle in $\bar{M}(c)$.
\end{abstract}

Keywords: Semi-Invariant Submanifolds, Semi-Invariant Product, Kenmotsu space form

Mathematics Subject Classification: 53C40

\section{Introduction}

The concept of CR-submanifold of a Kaehlerian manifold was first introduced by Bejancu [1] generalizing complex submanifolds and totally real submanifolds. Since then many papers had been published on this topic which is still the subject of much research. Bejancu and Papaghuic introduced and studied the notion of semi-invariant submanifolds of a Sasakian manifolds ([3] p. 101) which is a corresponding notion to that one of CR-submanifolds in a Kaehler manifold. Thus semi-invariant products are corresponding to CRproduct introduced by B. Y. Chen [5]. In fact, he obtained a necessary and sufficient condition for a CR-submanifold of a Kaehler manifold to be CRproduct in terms of the fundamental tensor of Weingarten.

On the other hand there are notions of circle and sphere in Riemannian geometry. The purpose of this note is to study semi-invariant product of a Kenmotsu space form by using the notion of circle.

Precisely we prove the following :

Theorem Let $M$ be a semi-invariant submanifold of a Kenmotsu space form $\bar{M}(c)(c \neq-1)$ such that every geodesic in $M$ is a circle in $\bar{M}(c)$. Then $M$ is a semi-invariant product in $\bar{M}(c)$. 


\section{Preliminaries}

Let $\bar{M}^{2 n+1}$ be an almost contact metric manifold with structure tensor $(\phi, \xi, \eta, g)$ where $\phi$ is a $(1,1)$ type tensor field, $\xi$ is a vector field, $\eta$ is 1 -form and $g$ is the associated Riemannian metric on $\bar{M}$. Then by definition [4], we have

$$
\begin{gathered}
\phi^{2} X=-X+\eta(X) \xi, \quad \eta(\xi)=1, \quad \phi \xi=0, \quad \eta(\phi)=0 \\
g(\phi X, \phi Y)=g(X, Y)-\eta(X) \eta(Y),
\end{gathered}
$$

for any vector fields $X$ and $Y$ tangent to $\bar{M}$.

In [8], Kenmotsu studied a new class of almost contact Riemannian manifold which satisfies the following condition :

$$
\left(\bar{\nabla}_{X} \phi\right)(Y)=g(\phi X, Y) \xi-\eta(Y) \phi X, \quad \bar{\nabla}_{X} \xi=X-\eta(X) \xi
$$

where $\bar{\nabla}$ denotes the Riemannian connection of $g$.

This structure is closely relative to the warped product of two Riemannian manifolds. Jansen and Vanhecke [7] called such manifolds Kenmotsu manifolds. One of the typical examples of Kenmotsu manifolds is the hyperbolic space $\bar{M}(-1)$.

Let $M$ be an $m$-dimensional Riemannian manifold isometrically immersed in Kenmotsu manifold $\bar{M}$ and suppose that the structure vector field $\xi$ of $\bar{M}$ is tangent to $M$.

Definition $A$ submanifold $M$ of $\bar{M}$ is called a semi-invariant submanifold if it is endowed with the pair of orthogonal distributions $D$ and $D^{\perp}$ satisfying the conditions :

(i) $T M=D \oplus D^{\perp} \oplus\{\xi\}$, where $\{\xi\}$ denotes the distribution spanned by $\xi$.

(ii) the distribution $D$ is invariant by $\phi$, that is, $\phi D_{x}=D_{x}$ for each $x \in M$.

(iii) the distribution $D^{\perp}$ is anti-invariant by $\phi$, that is, $\phi D_{x}^{\perp} \subset T_{x}^{\perp} M$ for each $x \in M$, where $T_{x}^{\perp} M$ is the normal space at $x \in M$.

Suppose that the dimension of $D$ (resp. $D^{\perp}$ ) be $2 p$ and $q$. When $p=0$ (resp. $q=0$ ) the semi-invariant submanifold $M$ is an anti-invariant submanifold (resp. an invariant submanifold).

Let $M$ be a semi-invariant submanifold of a Kenmotsu manifold $\bar{M}$. We say that $M$ is a semi-invariant product if it is locally a Riemannian product $M_{1} \times M_{2}$ where $M_{1}$ is an invariant-submanifold of $\bar{M}$ and $M_{2}$ is an anti-invariant submanifold of $\bar{M}$ such that the structure vector field $\xi$ of $\bar{M}$ is normal to $M_{2}$.

A Kenmotsu manifold $\bar{M}$ with constant $\phi$-holomorphic sectional curvature $c$ is called Kenmotsu space form and it is denoted by $\bar{M}(c)$. The curvature tensor of $\bar{M}(c)$ is given by $[10]$

$$
\bar{R}(X, Y) Z=\frac{c-3}{4}[g(Y, Z) X-g(X, Z) Y]+\frac{c+1}{4}[\eta(X) \eta(Z) Y-\eta(Y) \eta(Z) X
$$




$$
\begin{gathered}
+g(X, Z) \eta(Y) \xi-g(Y, Z) \eta(X) \xi+g(Z, \phi Y) \phi X \\
-g(Z, \phi X) \phi Y+2 g(X, \phi Y) \phi Z],
\end{gathered}
$$

for $X, Y, Z \in T \bar{M}$.

We shall denote by $\bar{\nabla}, \nabla, \nabla^{\perp}$ the Riemannian connections on $\bar{M}, M$ and the normal bundle respectively. They are related by

$$
\bar{\nabla}_{X} Y=\nabla_{X} Y+h(X, Y), \quad \bar{\nabla}_{X} N=-A_{N} X+\nabla_{X}^{\perp} N
$$

for all $X, Y \in T M, N \in T^{\perp} M$, where $h(X, Y)$ and $A_{N} X$ are the second fundamental forms satisfying

$$
g(h(X, Y), N)=g\left(A_{N} X, Y\right) .
$$

The Codazzi equation of $M$ is given by

$$
[\bar{R}(X, Y) Z]^{\perp}=\left(\bar{\nabla}_{X} h\right)(Y, Z)-\left(\bar{\nabla}_{Y} h\right)(X, Z),
$$

where $\left(\bar{\nabla}_{X} h\right)(Y, Z)=\nabla_{X}^{\perp} h(Y, Z)-h\left(\nabla_{X} Y, Z\right)-h\left(\nabla_{X} Z, Y\right)$,

for all vector fields $X, Y$ and $Z$ tangent to $M$.

An isotropic immersion is an isometric immersion such that all its normal curvature vectors have the same length at each point.

Moreover, we recall the notion of circle in a Riemannian manifold $\bar{M}$. A curve $x(t)$ of $\bar{M}$ parameterized by are length $t$ is called a circle if there exists a field of unit vector $Y_{t}$ along the curve which satisfies : $\bar{\nabla}_{t} X_{t}=K Y_{t}$ and $\bar{\nabla}_{t} Y_{t}=-K X_{t}$ for the tangent vectors $X_{t}=\dot{x}(t)$ where $K$ is a positive constant and $\bar{\nabla}_{t}=\bar{\nabla}_{\dot{x}(t)}$.

Now we state the following lemma for later use, the proof of which is quite similar to the case of Sasakian manifold ([3] p.106).

Lemma 2.1 A semi-invariant submanifold of a Kenmotsu manifold $\bar{M}$ is a semi-invariant product if and only if $A_{\phi D^{\perp}} D=0$.

Further we need the following lemma.

Lemma 2.2 [12] Let $M$ be a submanifold in a Riemannian manifold $\bar{M}$. Then the following two conditions are equivalent:

(i) the submanifold $M$ is nonzero isotropic and the second fundamental form $h$ of $M$ satisfies $\left(\bar{\nabla}_{X} h\right)(X, X)=0$ for all vector fields $X$ tangent to $M$.

(ii) every geodesic in $M$ is a circle in $\bar{M}$.

Remark Suppose every geodesic in $M$ is a circle in a Riemannian manifold $\bar{M}$, then the immersion is $\lambda$-isotropic i.e.

$$
g(h(X, X), h(X, X))=\lambda^{2} g(X, X) g(X, X), \quad \text { for all } \quad X \in T M
$$


which is equivalent to

$$
\begin{aligned}
& g(h(X, Y), h(W, Z))+g(h(X, Z), h(Y, W))+g(h(X, W), h(Y, Z)) \\
& =\lambda^{2}\{g(X, Y) g(W, Z)+g(W, Z) g(Y, W)+g(X, W) g(Y, Z)\}
\end{aligned}
$$

for all $X, Y, Z$ and $W$ tangent to $M$.

Now we prove the following lemma

Lemma 2.3 Let $M$ be a semi-invariant submanifold of a Kenmotsu space form $\bar{M}(c)$. Then the following are equivalent :

(i) $\left(\bar{\nabla}_{X} h\right)(X, X)=0$, for all $X \in T M$,

(ii) $\left(\bar{\nabla}_{X} h\right)(Y, Z)=\frac{c+1}{4}[g(X, \phi Y) \phi Z+g(X, \phi Z) \phi Y]^{\perp}$,

for all vector fields $X, Y$ and $Z$ tangent to $M$, where $\{*\}^{\perp}$ means the normal component of $\{*\}$.

Proof. Suppose (i) holds good. Then it can be easily seen that (i) is equivalent to

$$
\left(\bar{\nabla}_{X} h\right)(Y, Z)+\left(\bar{\nabla}_{Y} h\right)(Z, X)+\left(\bar{\nabla}_{Z} h\right)(X, Y)=0 .
$$

Now, from (2.7) we have

$$
\begin{gathered}
{[\bar{R}(X, Y) Z]^{\perp}=\left(\bar{\nabla}_{X} h\right)(Y, Z)-\left(\bar{\nabla}_{Y} h\right)(X, Z)} \\
=\frac{c+1}{4}[g(\phi Y, Z) \phi X-g(\phi X, Z) \phi Y+2 g(X, \phi Y) \phi Z]^{\perp} .
\end{gathered}
$$

Interchanging $Y$ and $Z$ we get

$$
\begin{gathered}
{[\bar{R}(X, Z) Y]^{\perp}=\left(\bar{\nabla}_{X} h\right)(Z, Y)-\left(\bar{\nabla}_{Z} h\right)(X, Y)} \\
=\frac{c+1}{4}[g(\phi Z, Y) \phi X-g(\phi X, Y) \phi Z+2 g(X, \phi Z) \phi Y]^{\perp} .
\end{gathered}
$$

Adding (2.9) and (2.11) we get (ii). The converse is trivial. $\diamond$

\section{Proof of the Theorem}

Let the geodesic in $M$ is a circle in $\bar{M}(c)(c \neq-1)$. Then by using the remark of section 2, the immersion is $\lambda$-isotropic. First we show that $\lambda$ is locally constant. To prove this, let us put $\lambda=\frac{\|h(X, X)\|}{\|X\|^{2}}$ and suppose that $x(t)$ be any geodesic with tangent vector $X_{t}$. Then $\lambda$ is constant along $x(t)$ because, by the assumptions (i) of Lemma 2.2, we have

$$
X_{t}\left(\lambda^{2}\right)=X_{t}\left(g\left(h\left(X_{t}, X_{t}\right) h\left(X_{t}, X_{t}\right)\right)=2 g\left(\bar{\nabla}_{X_{t}} h\right)\left(X_{t}, X_{t}\right), h\left(X_{t}, X_{t}\right)\right)=0,
$$


which shows that $\lambda$ is locally constant as the immersion is isotropic.

In order to prove our theorem, it suffices to show that $A_{\phi D^{\perp}} D=0$ i.e.

$$
g\left(\phi D^{\perp}, h(D, D)\right)=0 \quad \text { and } \quad g\left(\phi D^{\perp}, h\left(D, D^{\perp}\right)\right)=0,
$$

by using the Lemma 2.1 .

Now for making calculation simple, we assume that $\nabla X=\nabla Y=\nabla Z=$ $\nabla W=0$ at $p \in M$, and differentiating (2.8) with respect to an arbitrary vector field $U$, we have

$$
\begin{gathered}
g\left(\left(\bar{\nabla}_{U} h\right)(X, Y), h(Z, W)\right)+g\left(h(X, Y),\left(\bar{\nabla}_{U} h\right)(Z, W)\right)+g\left(\left(\bar{\nabla}_{U} h\right)(X, Z), h(Y, W)\right) \\
+g\left(h(X, Z),\left(\bar{\nabla}_{U} h\right)(Y, W)\right)+g\left(\left(\bar{\nabla}_{U} h\right)(X, W), h(Y, Z)\right) \\
+g\left(h(X, W),\left(\bar{\nabla}_{U} h\right)(Y, Z)\right)=0
\end{gathered}
$$

where we have used the fact that $\lambda$ is locally constant.

Now taking in particular $X \in D^{\perp}, U, Y, W, Z \in D$ in (3.1). Then from Lemma 2.3, we have

$$
\begin{gathered}
g(U, \phi Y) g(\phi X, h(Z, W))+g(U, \phi Z) g(\phi X, h(Y, W)) \\
+g(U, \phi W) g(\phi X, h(Y, Z))=0 .
\end{gathered}
$$

Putting $U=\phi W$ in (3.2) and using the fact that $\eta(X)=\eta(W)=\eta(Z)=0$, for $X \in D^{\perp}$ and $W, Z \in D$ and by virtue of the fact that $T M=D \oplus D^{\perp} \oplus\{\xi\}$ we get

$g(Y, W) g(\phi X, h(Z, W))+g(W, Z) g(\phi X, h(Y, W))+g(W, W) g(\phi X, h(Y, Z))=0$.

If $\operatorname{dim} D>2$, then by fixing $Y$ and $Z$ in (3.3), we can choose a vector field $W(\neq 0)$ in such a way that $g(Y, W)=g(W, Z)=0$. Then (3.3) gives

$$
g(\phi X, h(Y, Z))=0 \quad \text { at } p \quad \text { i.e. } \quad g\left(\phi D^{\perp}, h(D, D)\right)=0 .
$$

Next for $\operatorname{dim} D=2$, we put $Y=Z$ and choose a vector field $W(\neq 0)$ in such a way that $g(Y, W)=0$. Then (3.3) gives

$$
g(\phi X, h(Y, \phi Y))=0 \quad \text { at } p \quad \text { i.e. } \quad g\left(\phi D^{\perp}, h(D, D)\right)=0 .
$$

Finally putting $Z=\phi Y=\phi W$ in (3.3), we get

$$
g(\phi X, h(Y, \phi Y))=0 \quad \text { at } p \quad \text { i.e. } \quad g\left(\phi D^{\perp}, h(D, D)\right)=0 .
$$

Proceeding in the same way and using (3.1) and Lemma 2.3, we get

$$
g(\phi Z, h(X, W))=0 \quad \text { at } p \text { i.e. } \quad g\left(\phi D^{\perp}, h\left(D, D^{\perp}\right)\right)=0 .
$$


for $X, Z \in D^{\perp}, W(=U)=\phi Y \in D$, and which completes the proof.

\section{References}

[1] A. Bejancu, CR-Submanifold of a Kaehler manifold I, Proc. Amer. Math. Soc., 69 (1978), 135-142.

[2] A. Bejancu, CR-Submanifold of a Kaehler manifold II, Trans. Amer. Math. Soc., 250 (1979), 333-345.

[3] A. Bejancu, Geometry of CR-Submanifold, Mathematics and its Application, D. Reidel Publ. Co., 1986.

[4] D. E. Blair, Contact manifold in Riemannian geometry, Lecture Note in Math. Vol. 509, Springer Verlag, N. York, 1976.

[5] B. Y. Chen, CR-Submanifold of a Kaehler manifold I, J. Diff. Geometry, 16 (1981), 305-322.

[6] B. Y. Chen, Geometry of Submanifolds, Marcel Dekker, New York, 1973.

[7] D. Jansen and L. Vanhecke, Almost contact structure and curvature tensor, Kodai Math. J., 4 (1981), 1-27.

[8] K. Kenmotsu, A class of almost contact Riemannian manifold, Tohoku Math. J., 24 (1972), 73-103.

[9] M. Kobayashi, Semi-invariant submanifolds of a certain class of almost contact manifolds, Tensor (N. S.), 43 (1986), 28-36.

[10] K. Matsumoto, I. Mihai and M. H. Shahid, Certain submanifolds of a Kenmotsu manifold, The Third Pacific Rim Geometry Conference, Korea, 1996, 183-193.

[11] B. O. Neill, Isotropic and Kaehler immersion, Canad J. Math., 17 1965, 905-915.

[12] K. Nomizu, A characterization of the Veronese varieties, Nogaya Math. J., 60 (1976), 181-188.

[13] K. Nomizu and K. Yano, On circles and sphere in Riemannian geometry, Math. Ann., 21 (1974), 163-170.

Received: September 24, 2005 